
\title{
25 Research Soure \\ Quality of Life After Definitive Linac Stereotactic Radiotherapy for Prostate Cancer: Longitudinal Study
}

Hideomi Yamashita ( $\nabla$ yamashitah-rad@h.u-tokyo.ac.jp )

The University of Tokyo Hospital

Mami Ogita

The University of Tokyo Hospital

Subaru Sawayanagi

The University of Tokyo Hospital

Yuki Nozawa

The University of Tokyo Hospital

Osamu Abe

The University of Tokyo Hospital

\section{Research Article}

Keywords: Stereotactic body radiotherapy, prostate cancer, quality of life, patient-reported outcomes, radiation therapy

Posted Date: June 15th, 2021

DOI: https://doi.org/10.21203/rs.3.rs-583198/v1

License: (c) (1) This work is licensed under a Creative Commons Attribution 4.0 International License. Read Full License 


\section{Abstract}

Prostate cancer represents the second most common malignancy in the world and majority of patients have diagnosis of localized disease. We examined quality of life after stereotactic body radiation therapy (SBRT) for prostate cancer. We included patients treated between 2016 and 2020. Inclusion criteria were: adenocarcinoma of the prostate; class risks low, intermediate, and high; WHO performance status 02. QoL was measured using the Functional Assessment of Cancer Therapy - Prostate (FACT-P). 439 patients were included, treated with SBRT. Median age was 73. Median follow-up was 34 months. FACT P-TOI ( $p<0.0001)$, FACT G Total $(p=0.0003)$, and FACT P-total $(p<0.0001)$ declined at 1 month after the last SBRT, then recovered, and returned to the same level as before treatment at 3-4 month. The decrease in QoL at the first month was particularly remarkable in patients who received long-term hormone injections (36\% patients). One month after the end of SBRT, about $22 \%$ patients were "quite a bit" or more in trouble with any side effect. We showed the longitudinal changes of quality of life by FACT-P after SBRT for prostate cancer. Prostate SBRT appears to be overall well tolerated.

\section{Introduction}

Prostate cancer (PC) represents the second most common malignancy in the world and the majority of PC patients have diagnosis of localized disease (1). There are multiple, efficacious guidelinerecommended treatment options for localized PC, including radical prostatectomy, external beam radiation, and brachytherapy. A recent American Society for Radiation Oncology/American Society of Clinical Oncology/ American Urological Association (ASTRO/ASCO/AUA) guideline (2) included recommendations regarding use of stereotactic body radiotherapy (SBRT) for PC. According to the National Comprehensive Cancer Network (NCCN) Guideline Ver1. 2021 (3), 7.25-8 Gy * 5 radical SBRT for $\mathrm{PC}$ is indicated for low-risk to high- and ultra-high-risk cases. There is increasing awareness that quality of life alongside objective measures of late adverse genitourinary and gastrointestinal toxicity are essential to this decision-making process in the management of patients with PC. To the best of our knowledge, there have been no reports of evaluation of quality of life ( $Q \circ L)$ fluctuations after radical prostate SBRT using the Functional Assessment of Cancer Therapy-Prostate (FACT-P) so far. The present study investigated long-term QoL of patients after SBRT using a valid and self-administered QoL questionnaire of FACT-P.

\section{Materials And Methods}

We conducted a cohort prospective survey of patients affected by PC treated with SBRT at our institution (University of Tokyo Hospital). Inclusion criteria were: (1) histologically proven adenocarcinoma of the prostate; (2) NCCN class risks low, intermediate, or high; (3) World Health Organization (WHO) performance status 0-2. Exclusion criteria were: (1) lymph node metastasis; (2) bone metastasis; (3) castration-resistant prostate cancer; (5) after radical prostatectomy; (6) SBRT for local recurrence after external beam radiation therapy; (7) local recurrence after high-intensity focused ultrasound (HIFU); (8) 
not 5 fractionations. We didn't put a limit on prostate volume nor baseline International Prostate Symptom Score (IPSS) in order to include patients.

\section{Combination hormone therapy}

Basically, short-term hormones of 4-6 months were used in the medium-risk unfavorable group, and 1.52 years of hormone therapy were used in the high-risk group. There was no concomitant use of Bicalutamide, and the first Degarelix injection and the second and subsequent Leuprorelin injections. SBRT was performed when prostate-specific antigen (PSA) dropped to near $1.0 \mathrm{mg} / \mathrm{mL}$. However, SBRT was started by the second month in the short term and by half a year at the longest in the long-term administration group.

\section{SBRT method}

The irradiation dose was planning target volume (PTV) 95\% prescription, 36.25 Gy in 5 fractions before July 2018 was $54 \%$ and 40 Gy in 5 fractions after that was $43 \%$. SBRT was performed using volumetric modulated arc therapy (VMAT) with the use of flattening filter free beams using a linear accelerator with image-guidance. SBRT was performed 5 times every other day excluding weekends. There were many cases in which SpaceOAR was inserted before SBRT. See our past report (4) for spacer insertion, planning magnetic resonance imaging (MRI) and computed tomography (CT) scan for SBRT, and the definition of target volume and organs at risk. The patient was given Glycerin enema of 30 or $40 \mathrm{cc} 2$ hours before examination or SBRT. After that, the patients were asked to drink water and to store their urine for 2 hours. From 4 days before the examination to the last day of SBRT, the patients were encouraged to take Elobixibat Hydrate or Macrogol 4000 every day. SBRT dose constrains were shown in the Table 1.

\section{Follow-up}

Follow-up intervals were calculated from the date of last SBRT dose. Outpatient follow-up is conducted 1 month and 3-4 months after the end of SBRT, every 3 months until 2 years, and every 6 months after the 2nd year. We have asked patients to fill in a QoL questionnaire each follow-up time since May 2016. PSA is measured every time, and when it exceeds $2.0 \mathrm{ng} / \mathrm{mL}$ twice in a row, contrast-enhanced MRI and/or

${ }^{18} \mathrm{~F}$-fluorodeoxyglucose (FDG) positron emission tomography (PET) examination is added to check for recurrence. When treatment such as chemotherapy was started for other double cancers, it was excluded from the questionnaire survey.

\section{Statistical analysis}

The risk classification was based on the NCCN risk classification v1. 2021 for PC. The paired t test was used for comparison of baseline. All P-values were two-sided, with $p<0.05$ considered statistically significant. The FACT-P instrument (version 4) is a multidimensional, self-administered 39-item questionnaire. A FACT-P total score was obtained by adding emotional well-being (EWB), family wellbeing (FWB), physical well-being (PWB), social well-being (SWB) and prostate cancer subscale (PCS) (range 0-152), FACT-G (General) total score by adding EWB, FWB, PWB, SWB (range 0-104) and FACT-P 
Trial Outcome Index (TOI) by adding FWB, PWB and PCS subscale scores (range 0-104) version 4 of the FACT scoring guidelines (5). Prorating was carried out only if more than $50 \%$ of the items on each subscale were answered. For all scales, the higher the score was, the better the QOL was. This study was approved by the institutional review board of Tokyo University (No. 3372-6). All methods were carried out in accordance with relevant guidelines and regulations. Informed consent was obtained from all subjects.

\section{Results}

Baseline patient characteristics are shown in Table 2. Median follow-up was 34 months (maximum: 54 months). Between May 2016 and Dec 2020, 439 patients answered the FACT-QoL questionnaire at some point. These 439 patients were all that had been treated with SBRT for prostate cancer with a curative intent in our institution during this period. The median age was 73 years, the median PSA before all treatments was $9.2 \mathrm{ng} / \mathrm{mL}$, clinical T2a was the highest at 47\%, and Gleason Score Group by NCCN was $28 \%$ and $27 \%$ for Group 2 and 3, respectively. The NCCN medium risk was $53 \%$ and high risk was also included at $42 \%$. Short-term hormone combination for $4-6$ months was $42 \%$ and long-term hormone combination was $36 \% .177$ patients $(40 \%)$ had hydrogel spacer inserted between the prostate and rectum before SBRT.

The cumulative total number of patients who were caught in exclusion criteria during the same period was: SBRT for (1) lymph node metastasis in 12 patients; (2) bone metastasis in 11 patients; (3) castration-resistant prostate cancer in seven patients; (5) after radical prostatectomy in one patient; (6) local recurrence after external beam radiation therapy in two patients; (7) local recurrence after highintensity focused ultrasound (HIFU) in three patients; (8) not 5 fractionations in one patient.

314 patients (71\%) before SBRT, 242 patients (55\%) at 1 month, 46 patients at 2 month, and 246 patients at 3 to 4 month, 197 patients at 5-7 month, 190 patients at 8-10 month, 165 patients at 11-13 month, 137 patients at 14-16 month, 117 patients at 17-19 month, 102 patients at 20-22 month, 85 patients at 23-25 month, 61 patients at 29-31 month, 50 patients at 32-34 month, 54 patients at 35-37 month, 44 patients at 38-42 month, 38 patients at 43-54 month, 29 patients at 43-48 month, 9 patients at 49-54 month filled out the questionnaire. Since we included all patients received SBRT in this survey, approximate $30 \%$ of patients did not complete the questionnaire before treatment.

In all of FACT P-TOI, FACT G Total, and FACT P-total, the QoL score at 1 month after the end of SBRT was once significantly lower than the value before SBRT (Fig. 1). The average score for each QoL dropped from 78.84 (standard error: 0.77 ) before SBRT to 72.35 (1.02) at 1 month in FACT P-TOI ( $p<0.0001$ by ttest), from $79.91(0.79)$ to $75.38(0.96)$ in FACT G Total $(p=0.0003)$, and from $114.22(1.05)$ to 105.78 (1.31) in FACT P-total $(p<0.0001)$, respectively. After that, an improvement trend was seen in the second month (the average score: 74.62 (SE: 2.57), 77.13 (2.09), and 107.88 (3.23), respectively). Furthermore, at 3-4 month, all of FACT P-TOI ( $p=0.0011$, the average score: 76.80 (SE: 0.88)), FACT G Total $(p=0.0410$, $78.15(0.94))$, and FACT P-total $(p=0.0029,111.19(1.24))$ improved significantly from the 1 st month. In the subsequent course, there was basically no re-decrease in the plateau until the 49-54th months. 
In the long-term hormone-administered group, the QOL reduction of FACT P-TOI (67.99) and FACT P-total (102.08) at 1 month was larger than that in the short-term administration group (administration period of 6 months or less) and the hormone-free group (Fig. 2). In the hormone-free group, the bottom of all QoL was 2 months instead of 1 month. With respect to use of hydrogel spacer, the score of FACT P-TOI, FACT G Total, and FACT P-total at 1 month was 71.35 (SE: 1.02), 74.41 (0.96), and 104.26 (1.31) without spacer versus $74.97(1.76)(p=0.11$ by unpaired t-test), $77.94(1.69)(p=0.10)$, and $109.74(2.28)(p=0.062)$ with spacer, respectively. With respect to the SBRT dose, the score of FACT P-TOI, FACT G Total, and FACT Ptotal at 1 month was 72.58 (SE: 1.17), 75.22 (1.09), and 105.60 (1.46) after $36.25 \mathrm{~Gy}$ ( $p=0.33$ of 36.25 Gy versus $40 \mathrm{~Gy}, \mathrm{p}=0.68$ of 36.25 Gy versus $42.5 \mathrm{~Gy}$, and $\mathrm{p}=0.99$ of $40 \mathrm{~Gy}$ versus $42.5 \mathrm{~Gy}$ ), and 71.77 (2.21), 76.05 (2.16), and 106.39 (3.02) ( $p=0.71,0.82$, and 0.74) after $40 \mathrm{~Gy}, 71.85$ (6.29), 74.15 (5.47), and $105.15(7.88)(p=0.79,0.84$, and 0.88$)$ after $42.5 \mathrm{~Gy}$, respectively.

P7 (I have difficulty urinating) (the average score: 3.25 before SBRT, 2.49 at 1 month, and 3.14 at 2 months) and BL2 (I urinate more frequently than usual) $(2.58,1.62$, and 2.22 ) bottomed out one month after the end of SBRT (both $p<0.0001$ ) (Fig. 3). Improvement was seen at 2 month and was fully recovered at 3-4 month. P6 (I have trouble moving my bowels) and P8 (My problems with urinating limit my activities) on restriction of activity by urine continued to decline until the second month and recovered at 3-4 month (Fig. 3).

The percentage of those who answered that they were suffering from side effects of GP5 (I am bothered by side effects of treatment) was also the highest at $22 \%$ in the first month after the end of SBRT from $8 \%$ at pre-SBRT, and dropped to $11 \%$ in the 3rd to 4 th months (Fig. 4). After that, it gradually decreased over time. Including "somewhat", it changed from $15 \%$ before SBRT into $45 \%$ at 1 st month, $32 \%$ at 2 nd month, and $28 \%$ at $3-4$ months.

\section{Discussion}

In this prospective study, we evaluated the QoL of 439 patients treated with SBRT for low, intermediate, and high risk PC. Most of the QoL evaluations using FACT-P after SBRT for PC so far are only after SBRT for metastatic PC (6-8). Since they did not irradiate the primary prostate lesion, it cannot be compared with the QoL after the radical SBRT this time. This time, using FACT-P questionnaire, it is highly novel to see the time-series changes in QoL after radical PC SBRT. In this report, QoL declined at 1 month after the last SBRT, then recovered, and returned to the same level as before treatment 3-4 months. The decrease in QoL at the first month was particularly remarkable in patients who received combined long-term hormone injections. One month after the last SBRT, about $22 \%$ of people were "quite a bit or very much" in trouble with any side effect. Since the QOL score of patient-reported side effects (i.e. P6, P7, P8, and BL2) was also declining at the same time this time, this QOL changes in chronological order would be for SBRT-induced acute toxicity, though we did not compare patient-reported outcome measures (PROMS) with toxicity assessment like common terminology criteria for adverse events (CTCAE). In this study, we can't report data on correlation between physician- and patient-reported outcomes. 
Chen et al. (6) treated 100 PC patients with $36.25 \mathrm{~Gy}$ in five fractions (7.25 Gy per fraction) with a median follow-up of 27 months. Biochemical disease-free survival was $99 \%$, and no acute Grade 3-5 gastrointestinal (GI) or genitorurinary (GU) toxicity was recorded (6). According to Franzese C (10), acute rectal toxicity of moderate-severe grade is reported in $6 \%$ patients after SBRT. Grade 2-3 GI toxicity was reported in 2 (2\%) patients after SBRT (10). Acute GU toxicity of grades 2 and 3 was $31 \%$ after SBRT (10). The evidence from the phase 3 trial (HYPO-RT-PC) (17) suggested higher patient-reported toxicity with ultra-hypofractionation of $6.1 \mathrm{~Gy} \times 7$ fractions. Grade 2 or worse acute toxicity estimates for ultrahypofractionation were similar to standard fractionation, ranging from $4-24 \%$ for GI toxicity and $4-40 \%$ for GU toxicity (17). By contrast, the results from the randomized PACE-B trial (16) suggested that substantially shortening treatment courses with SBRT did not increase either GI or GU acute toxicity. Worst acute RTOG GI and GU toxic effect proportions of grade 2 or more were $43(10 \%)$ and $96(23 \%)$ of 415 patients after SBRT, respectively (16). In our study, 45\% patients answered "somewhat" or more and $22 \%$ "quite a bit" or more in GP5 (I am bothered by side effects of treatment) at 1 month after the last SBRT. In general, patient-reported side effects are more frequent than doctor-judged side effects.

According to Franzese $C(10)$, regarding the late setting, grades 2 and 3 rectal toxicity was $1 \%$ and $0 \%$ after SBRT. While GU side-effect of Grade 1 was $44 \%$, grades 2 and 3 toxicity was $2 \%$ and $0 \%$ after SBRT (10). According to the review of Wang et al. (11), after SBRT for PC, most toxicities were grade 1-2 with a very low overall incidence of grade $3-5$ toxicity (usually $<3 \%$ ). According to Hwang et al. (12), after SBRT with periprostatic hydrogel spacer (SpaceOAR; Augmenix) for localized PC, no grade 3-5 GU or GI toxicity was recorded. New grade 2 GU toxicity (urgency or dysuria) was present in $30 \%$ of patients at 1 month and in $12 \%$ of patients at 1 year post-treatment and GI toxicity was limited to grade 1 (16\%) during SBRT, although $4 \%$ of patients developed grade 2 during the first 4 weeks after SBRT (12). Periprostatic hydrogel placement followed by prostate SBRT may result in minimal GI toxicity. In our study, $10-30 \%$ and $0-13 \%$ of patients answered "somewhat" or more and "quite a bit" or more in GP5 after 3-4 month, respectively.

There were times when the number of questionnaires was small, such as the 2 nd month and after the 38th month. Since the number of questionnaires was very small of 10.5\% (46/439 patients) at 2 month, the statistical power might also be low. Since we included all patients treated with SBRT during this period, only $71 \%$ patients had a baseline assessment and $55 \%$ was evaluated at 1 month. In this way, our QOL assessments might have become a heterogeneous distribution.

With respect to the previous report of the QoL change after the conventional IMRT method, only reports of changes in QoL after the conventional IMRT method reported that the timing of the initial questionnaire was 3 months after irradiation and no decrease in QoL was observed (13-15). This time, in addition to before SBRT, we collected QoL data for the earlier time of the first and second months after SBRT, which is also highly novel. We compared our findings with the previous QOL data of patients treated using low dose rate (LDR) and high dose rate (HDR) Brachytherapy. According to Slevin F et al. (18), maximal deterioration in mean urinary $(p<0.001)$ and sexual summary scores using a validated expanded prostate cancer index composite (EPIC) questionnaire was noted 6 weeks after implant, with severe 
urinary symptoms and moderate bowel/sexual symptoms after HDR brachytherapy. At 6 months, urinary and bowel QOL had improved to mild impairment, which then fully resolved at 10 months (18). According to Strom TJ et al. (19), after median follow-up of 32 months, HDR brachytherapy (27-28 Gy in two fractions) and IMRT (74-81 Gy in 37-45 fractions) patients had significantly less deterioration in their urinary HRQOL than LDR ( $145 \mathrm{~Gy}$ in one fraction) brachytherapy patients at 1 and 3 months after irradiation. The only significant decrease in bowel HRQOL between the groups was seen 18 months after treatment, at which point IMRT patients had a slight, but significant, deterioration in their bowel HRQOL compared with HDR and LDR brachytherapy patients (19). As with Brachytherapy, the dose was prescribed inhomogeneous in order to spare the urethra. The homogeneous dose distribution may affect patient in a different way than with a heterogeneous brachytherapy treatment plan.

According to the Georgetown University's report (6), which is the only one looking at the QoL one month after SBRT, a median baseline American Urological Association Symptom Score (AUA) symptom score of 8 significantly increased to 11 at 1 month $(p=0.001)$, however returned to baseline at 3 months $(p=$ $0.60)$.

\section{Conclusions}

QoL declined at 1 month after the end of prostate SBRT, then recovered, and returned to the same level as before treatment 3-4 months after SBRT. The decrease in QoL at the first month was particularly remarkable in patients who received long-term hormone injections. One month after the end of SBRT, about $22 \%$ of people were "quite a bit or very much" in trouble with some side effects.

\section{References}

1. Schröder FH, Hugosson J, Roobol MJ, Tammela TLJ, Ciatto S, Nelen V, et al. Screening and prostatecancer mortality in a randomized European study. N Engl J Med. 2009; 360: 1320-1328.

2. Morgan SC, Hoffman K, Loblaw DA, et al. Hypofractionated Radiation Therapy for Localized Prostate Cancer: Executive Summary of an ASTRO, ASCO and AUA Evidence-Based Guideline. J Urol. 2019 Mar; 201(3): 528-534.

3. https://www.nccn.org/professionals/physician_gls/pdf/prostate.pdf

4. Ogita M, Yamashita H, Sawayanagi S, Takahashi W, Nakagawa K. Efficacy of a hydrogel spacer in three-dimensional conformal radiation therapy for prostate cancer. Jpn J Clin Oncol. 2020; 50: 303309 .

\section{5. https://www.facit.org/measures/FACT-P}

6. Chen LN, Suy S, Uhm S, Oermann EK, Ju AW, Chen V, et al. Stereotactic body radiation therapy (SBRT) for clinically localized prostate cancer: the Georgetown University experience. Radiat Oncol. 2013; 8: 58.

7. McKay R, Haider B, Duh MS, et al. Impact of symptomatic skeletal events on health-care resource utilization and quality of life among patients with castration-resistant prostate cancer and bone 
metastases. Prostate Cancer Prostatic Dis. 2017 Sep; 20(3): 276-282.

8. Saad F, Ivanescu C, Phung D, et al. Skeletal-related events significantly impact health-related quality of life in metastatic castration-resistant prostate cancer: data from PREVAIL and AFFIRM trials. Prostate Cancer Prostatic Dis. 2017 Mar; 20(1): 110-116.

9. Joseph KJ, Alvi R, Skarsgard D, et al. Analysis of health related quality of life (HRQoL) of patients with clinically localized prostate cancer, one year after treatment with external beam radiotherapy (EBRT) alone versus EBRT and high dose rate brachytherapy (HDRBT). Radiat Oncol. 2008 Jul 15; 3: 20.

10. Franzese C, D'agostino G, Brina LD, et al. Linac-based stereotactic body radiation therapy vs moderate hypofractionated radiotherapy in prostate cancer: propensity-score based comparison of outcome and toxicity. Br J Radiol. 2019 May; 92(1097): 20190021.

11. Wang K, Mavroidis P, Royce TJ, Falchook AD, Collins SP, Sapareto S, Sheets NC, Fuller DB, El Naqa I, Yorke E, Grimm J, Jackson A, Chen RC. Prostate Stereotactic Body Radiation Therapy: An Overview of Toxicity and Dose Response. Int J Radiat Oncol Biol Phys. 2020 Dec 22:S0360-3016(20)34356-X.

12. Hwang ME, Mayeda M, Liz M, et al. Stereotactic body radiotherapy with periprostatic hydrogel spacer for localized prostate cancer: toxicity profile and early oncologic outcomes. Radiat Oncol. 2019 Aug 2; 14(1): 136.

13. Kretschmer A, Ploussard G, Heidegger I, et al. Health-related Quality of Life in Patients with Advanced Prostate Cancer: A Systematic Review. Eur Urol Focus. 2020 Feb 20: S24054569(20)30064-X.

14. Feigenberg SJ, Lee WR, Desilvio ML, et al. Health-related quality of life in men receiving prostate brachytherapy on RTOG 98-05. Int J Radiat Oncol Biol Phys. 2005 Jul 15; 62(4): 956-964.

15. Wilkins A, Mossop H, Syndikus I, et al. Hypofractionated radiotherapy versus conventionally fractionated radiotherapy for patients with intermediate-risk localised prostate cancer: 2-year patientreported outcomes of the randomised, non-inferiority, phase $3 \mathrm{CHHiP}$ trial. Lancet Oncol. 2015 Dec; 16(16): 1605-1616.

16. Brand $\mathrm{DH}$, Tree AC, Ostler $\mathrm{P}$, et al. Intensity-modulated fractionated radiotherapy versus stereotactic body radiotherapy for prostate cancer (PACE-B): acute toxicity findings from an international, randomised, open-label, phase 3, non-inferiority trial. Lancet Oncol. 2019 Nov; 20(11): 1531-1543.

17. Widmark A, Gunnlaugsson A, Beckman L, et al. Ultra-hypofractionated versus conventionally fractionated radiotherapy for prostate cancer: 5-year outcomes of the HYPO-RT-PC randomised, noninferiority, phase 3 trial. Lancet. 2019 Aug 3; 394(10196): 385-395.

18. Slevin F, Sethugavalar B, Al-Qaisieh B, et al. Ten-year longitudinal health-related quality of life following iodine-125 brachytherapy monotherapy for localized prostate cancer. J Contemp Brachytherapy. 2020 Dec; 12(6): 540-546.

19. Strom TJ, Cruz AA, Figura NB, et al. Health-related quality-of-life changes due to high-dose-rate brachytherapy, low-dose-rate brachytherapy, or intensity-modulated radiation therapy for prostate cancer. Brachytherapy. Nov-Dec 2015; 14(6): 818-825. 


\section{Tables}

Table 1. Dose constraint of $40 \mathrm{~Gy}$ in 5 fractions

\begin{tabular}{|c|c|c|c|c|c|}
\hline \multicolumn{2}{|c|}{ Dose constraint } & \multicolumn{2}{|c|}{ With SpaceOAR } & \multicolumn{2}{|c|}{ Without SpaceOAR } \\
\hline Prostate & Volume & Goal & Torelance & Goal & Torelance \\
\hline Urethra & $\begin{array}{l}\operatorname{Max} \\
\operatorname{Max}\end{array}$ & & & & \\
\hline \multirow[t]{3}{*}{ PTV } & V110\% & $<0.5 \%$ & $<2 \%$ & $<0.5 \%$ & $<2 \%$ \\
\hline & $\begin{array}{c}\mathrm{D} 99 \% \\
\operatorname{Max}\end{array}$ & $\begin{array}{l}>39.5 \mathrm{~Gy} \\
<43.26 \mathrm{~Gy}\end{array}$ & $\begin{array}{l}>39.17 \mathrm{~Gy} \\
<44.14 \mathrm{~Gy}\end{array}$ & $\begin{array}{l}>39.2 \mathrm{~Gy} \\
<43.9 \mathrm{~Gy}\end{array}$ & $\begin{array}{l}>38.1 \mathrm{~Gy} \\
<45.5 \mathrm{~Gy}\end{array}$ \\
\hline & Mean & $<41.16 \mathrm{~Gy}$ & $<41.71 \mathrm{~Gy}$ & $<41.5 \mathrm{~Gy}$ & $<42 \mathrm{~Gy}$ \\
\hline \multirow[t]{4}{*}{ Bladder } & V40Gy & $<3.7 \%$ & $<6 \%$ & $<4.6 \%$ & $<6 \%$ \\
\hline & V20Gy & $<22.8 \%$ & $<35 \%$ & $<27.4 \%$ & $<49 \%$ \\
\hline & V40.83Gy & $<6.2 \mathrm{cc}$ & $<8 \mathrm{cc}$ & $<6.9 \mathrm{cc}$ & $<10 c c$ \\
\hline & $\begin{array}{c}\text { Max } \\
\text { Mean }\end{array}$ & $\begin{array}{l}<42.7 \mathrm{~Gy} \\
<11.92 \mathrm{~Gy}\end{array}$ & $\begin{array}{l}<44.14 \mathrm{~Gy} \\
<16.55 \mathrm{~Gy}\end{array}$ & $\begin{array}{l}<43.4 \mathrm{~Gy} \\
<13.7 \mathrm{~Gy}\end{array}$ & $\begin{array}{l}<45 \text { Gy } \\
<20 \text { Gy }\end{array}$ \\
\hline \multirow[t]{8}{*}{ Rectum } & & 1 & $\mathrm{~m}$ above anc & below the & \\
\hline & V40Gy & $<0.5 \%$ & $<1.5 \%$ & $<2.5 \%$ & $<4 \%$ \\
\hline & V36Gy & $<2.5 \%$ & $<5.7 \%$ & $<8 \%$ & $<11 \%$ \\
\hline & V32Gy & $<5 \%$ & $<10 \%$ & $<13 \%$ & $<17 \%$ \\
\hline & V30Gy & $<6.8 \%$ & $<13 \%$ & $<16 \%$ & $<20 \%$ \\
\hline & V20Gy & $<28.2 \%$ & $<34 \%$ & $<32 \%$ & $<39 \%$ \\
\hline & $\operatorname{Max}$ & $<40.83$ Gy & $<42.48 \mathrm{~Gy}$ & $<42.7 \mathrm{~Gy}$ & $<43.9$ Gy \\
\hline & Mean & $<16.88 \mathrm{~Gy}$ & $<18.21 \mathrm{~Gy}$ & $<17.4 \mathrm{~Gy}$ & $<19.6 \mathrm{~Gy}$ \\
\hline $\begin{array}{l}\text { Femoral head } \\
\text { Sigmonid colon }\end{array}$ & $\begin{array}{c}\text { Max } \\
\text { V30Gy }\end{array}$ & $\begin{array}{c}<17.66 \mathrm{~Gy} \\
<0 \mathrm{cc}\end{array}$ & $<18.76 \mathrm{~Gy}$ & $\begin{array}{c}<17.66 \mathrm{~Gy} \\
<1 \mathrm{cc}\end{array}$ & $<18.76 \mathrm{~Gy}$ \\
\hline Penile bulb & $\operatorname{Max}$ & $<40 \mathrm{~Gy}$ & & $<40$ Gy & \\
\hline Small bowel & V30Gy & $0 c c$ & $<5 c c$ & $0 c c$ & $<5 c c$ \\
\hline
\end{tabular}

Table 2. Patient characteristics 


\begin{tabular}{|c|c|c|}
\hline Factors & $\mathrm{N}$ & $\%$ \\
\hline Total & 439 & 100 \\
\hline \multicolumn{3}{|l|}{ Age (y ears old) } \\
\hline Median (range) & \multicolumn{2}{|c|}{73 (33-92) } \\
\hline Quartile & \multicolumn{2}{|c|}{68 and 78} \\
\hline \multicolumn{3}{|l|}{ iPSA (ng/mL) } \\
\hline Median (range) & \multicolumn{2}{|c|}{$9.2(1.6-24.0)$} \\
\hline Quartile & \multicolumn{2}{|c|}{6.3 and 14.7} \\
\hline \multicolumn{3}{|l|}{ Clinical T-stage } \\
\hline T1c & 84 & 19.1 \\
\hline T2a & 208 & 47.4 \\
\hline T2b & 20 & 4.6 \\
\hline T2c & 61 & 13.9 \\
\hline T3a & 46 & 10.5 \\
\hline T3b & 14 & 3.2 \\
\hline T4 & 6 & 1.4 \\
\hline \multicolumn{3}{|l|}{ Gleason score group } \\
\hline Group 1 & 46 & 10.5 \\
\hline Group 2 & 121 & 27.6 \\
\hline Group 3 & 117 & 26.7 \\
\hline Group 4 & 82 & 18.7 \\
\hline Group 5 & 73 & 16.6 \\
\hline \multicolumn{3}{|l|}{ Risk group } \\
\hline Low & 21 & 4.8 \\
\hline Intermediate-low & 84 & 19.1 \\
\hline Intermediate-high & 149 & 33.9 \\
\hline High & 112 & 25.5 \\
\hline Ver-high & 73 & 16.6 \\
\hline \multicolumn{3}{|l|}{ Hormonal therapy } \\
\hline None & 98 & 22.3 \\
\hline Short term & 183 & 41.7 \\
\hline Long term & 158 & 36.0 \\
\hline \multicolumn{3}{|l|}{ RT total dose } \\
\hline 36.25 Gy & 239 & 54.4 \\
\hline 40 Gy & 187 & 42.6 \\
\hline $42.5 \mathrm{~Gy}$ & 13 & 3.0 \\
\hline
\end{tabular}

Abbreviation: PSA = prostate-specific antigen, $\mathrm{RT}=$ radiation therapy

\section{Figures}




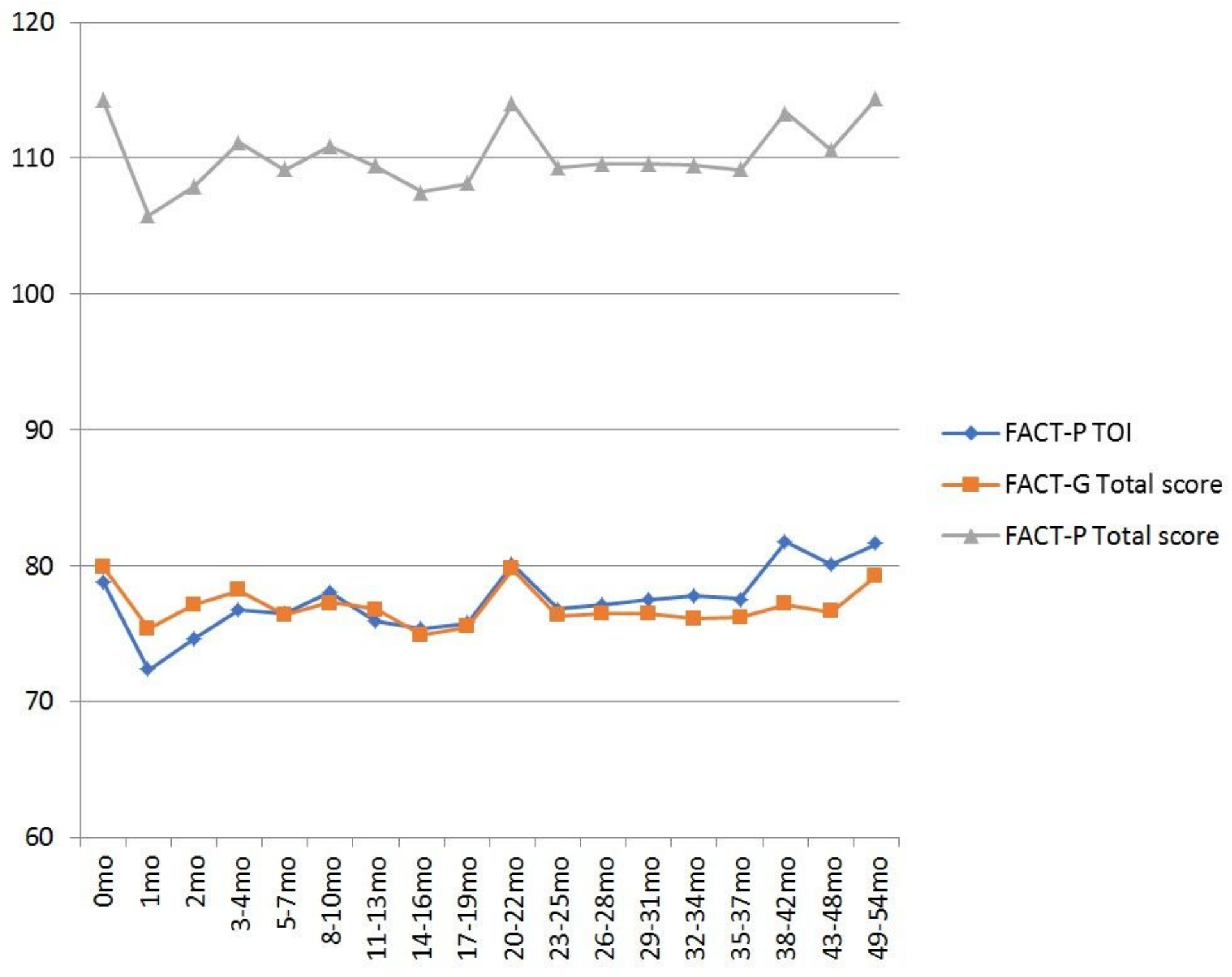

Figure 1

Mean Functional Assessment of Cancer Therapy-Prostate (FACT P) Trial Outcome Index score (TOI, blue line), Functional Assessment of Cancer Therapy-General (FACT G) Total score (orange line), and FACT Ptotal score (gray line) score. Time from last SBRT dose (months). 


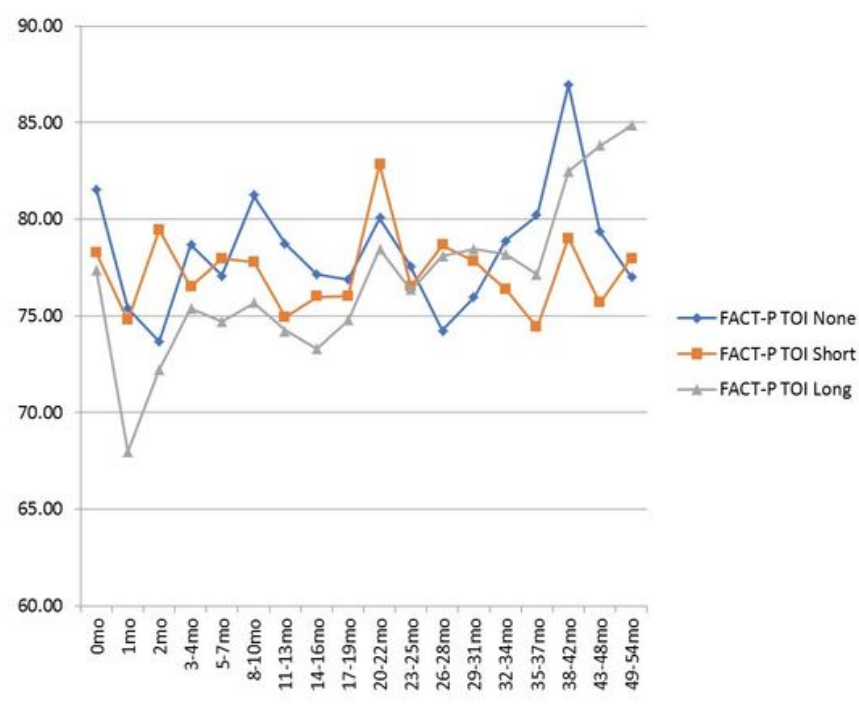

$2 a$

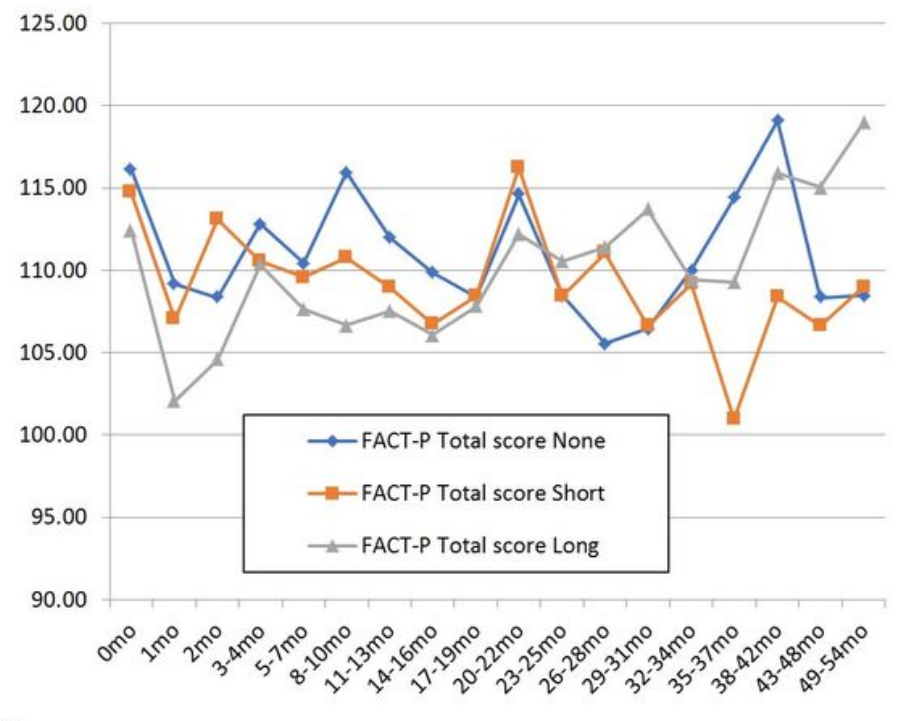

$2 b$

\section{Figure 2}

Mean Functional Assessment of Cancer Therapy-Prostate (FACT P) Trial Outcome Index (TOI) score (2-a) and mean FACT P-total score (2-b) by none (blue line), short term (orange line), and long term (gray line) hormonal therapy.

4.00

3.50

3.00

2.50

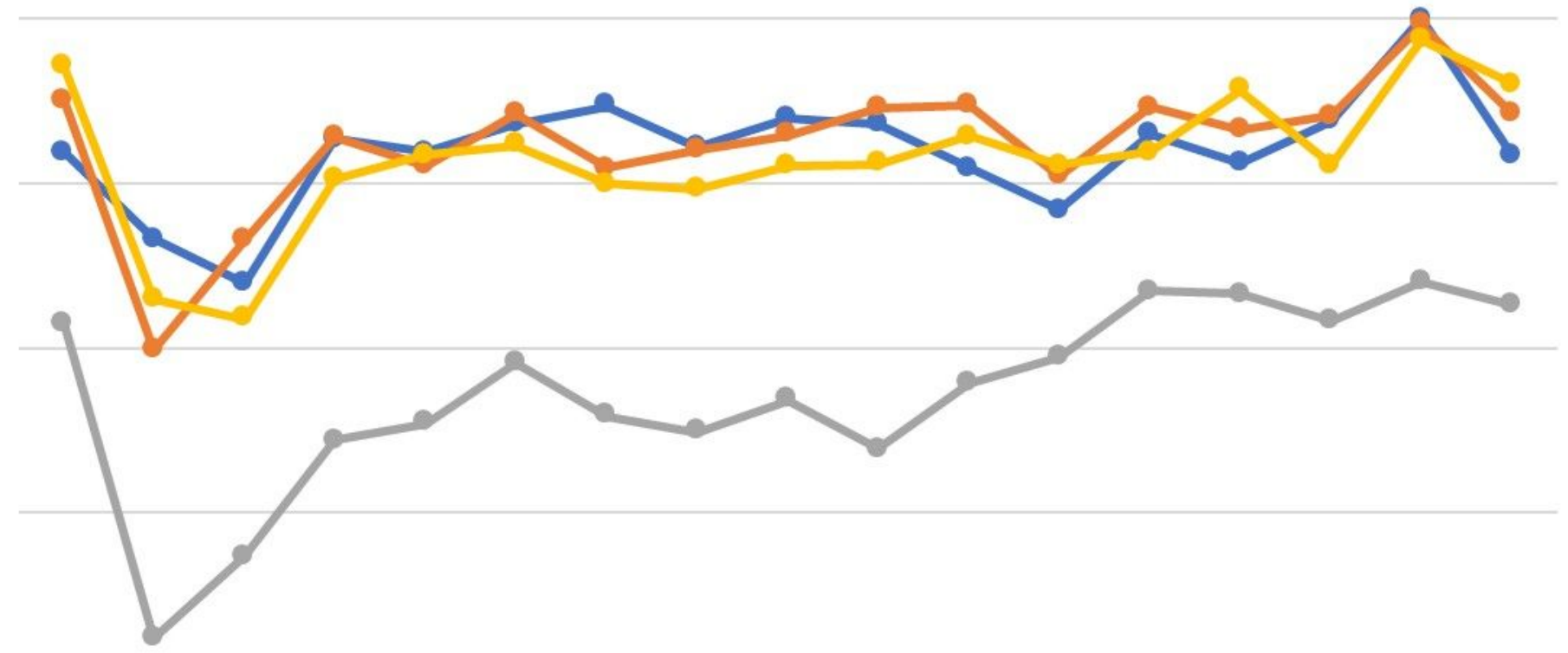

1.50

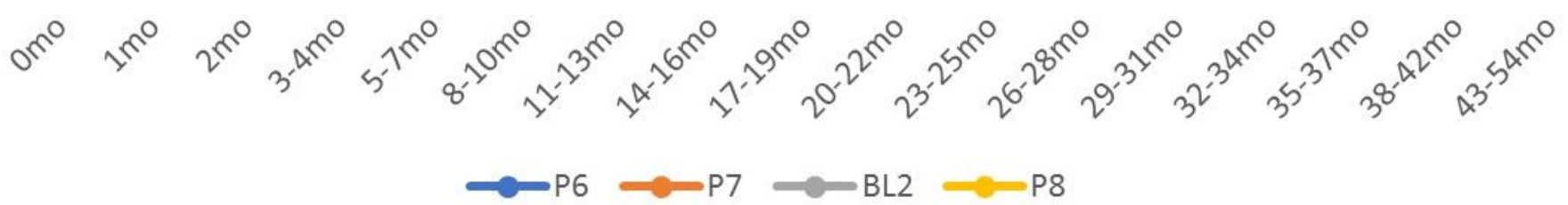


Figure 3

Mean four subscale scores. P6 (I have trouble moving my bowels): blue, P7 (I have difficulty urinating): orange, BL2 (I urinate more frequently than usual): gray, and P8 (My problems with urinating limit my activities): yellow line.

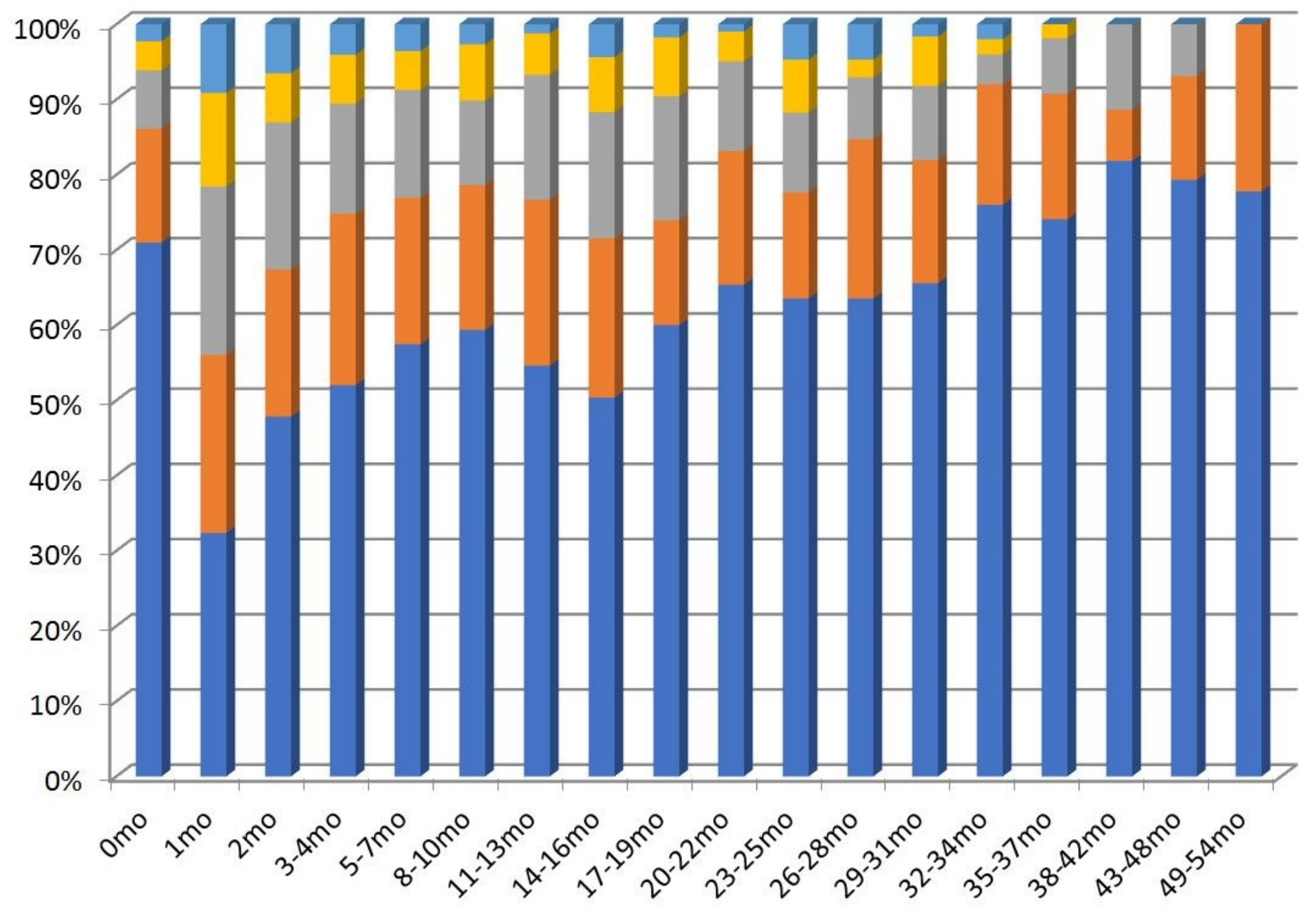

Figure 4

The distribution of answer to subscale GP5 (I am bothered by side effects of treatment) question (not at all: dark blue, a little bit: orange, somewhat: gray, quite a bit: yellow, and very much: sky blue). 